\title{
Digitale Identität - Zwischen Mythos und Wirklichkeit
}

Zu den sensibelsten Objekten im Netz gehört die digitale Identität. Nicht nur, dass sie die physische Identität unvollkommen und widersprüchlich abbildet, sie ist auch ein Hauptangriffsziel von Hackern und Kriminellen. Mit entwendeten Identitätsdaten lässt sich Geld verdienen und Vertrauen in geschäftliche und private Anwendungen zerstören.

Die natürliche Identität eines Menschen erscheint als einmalig und unverwechselbar. Sie wird anhand charakteristischer Identitätsattribute - z. B. durch körperliche Merkmale wie Erscheinungsbild und Verhalten - geprägt und so einem Gegenüber (wieder-) erkennbar. Durch eindeutige Namensgebung und Registereintrag wird diese Identität (über den Handschlag hinaus) rechtsfähig. Zum Nachweis einer behaupteten Identität gegenüber Fremden kann letztendlich ein vertrauenswürdiges - z.B. ein hoheitliches - Dokument dienen, auf dem individuelle Identitätsattribute vermerkt sind. Selbstverständlich existiert auch in diesem Szenario ein Spektrum von Fälschungs- und Betrugsmöglichkeiten.

In der digitalen Welt ist die Zuordnung von Personen zu einem geschäftsrelevanten Vorgang - im Allgemeinen unter der Bedingung, dass sich die Geschäftspartner nicht kennen - ebenfalls zwingend erforderlich. Wenn sie online Geld überweisen oder einkaufen, sich in Foren, sozialen Netzwerken oder E-Mail-Accounts einloggen, authentisieren sich Nutzer mit unterschiedlichen Verfahren. Auch hier greifen Mechanismen, die individuelle Attribute einer bestimmten Person zuordnen. Ein simples Beispiel ist die Verbindung von Benutzername und Passwort: Der (oft selbstgegebene) Benutzername gehört einer Person, die sich mit dem Passwort authentisiert. Eine so definierte digitale Identität ist jedoch weder einmalig noch unverwechselbar und üblicherweise an eine bestimmte Anwendung gebunden. Sie bleibt ohne einen Rechtsanker - z.B. ein Register über ein hoheitliches Dokument - lediglich partiell.

Bereits im Signaturgesetz hatte die Registrierung des Benutzers eine hervorgehobene Bedeutung: die Erstellung eines qualifizierten Zertifikats erfordert eine „zuverlässige“ Identifikation des Antragstellers durch den Zertifizierungsdiensteanbieter, die in der Regel allerdings auf nicht-elektronischem Wege durchgeführt wird und dabei meist auf die o.g. hoheitlichen Dokumente zurückgreift. Der seit 2011 ausgegebene neue Personalausweis würde über die dort implementierte eID-Funktion eine digitale Authentifikation erlauben und würde den oben beschriebenen Medienbruch vermeiden. Der mögliche Einsatz zur Registrierung nach dem Signaturgesetz ist nur als ein Beispiel zu verstehen, andere Anwendungen wie z.B. das Login zu kostenpflichtigen Diensten könnten dies ebenfalls nutzen. Warum findet dies aber nicht statt? An der Sicherheit und Zuverlässigkeit liegt es nicht. Diese steht außer Frage, für das zugehörige PACE Protokoll wurde der Beweis der formalen Korrektheit erbracht. Die kom- plizierte Infrastruktur auf Seiten des Nutzers aber auch eines Anbieters, der die eID-Funktion in sein Online Angebot integrieren will, stellt sicherlich ein großes Hindernis für eine breitere Nutzung dar ${ }^{1}$.

Anders sieht es aus, wenn Authentifikationsinstrumente verwendet werden müssen. Im Online-Banking ist eine große Anzahl davon bekannt: mTAN, SmartTAN, chipTAN, HBCI, um nur einige zu nennen. Hierfür werden in aller Regel zusätzliche Geräte benötigt, mit deren Hilfe eine TAN zu Absicherung der Transaktion generiert wird. Die Verfahren sind nicht einheitlich ${ }^{2}$ und werden von der kontoführenden Bank dem Kunden vorgegeben, sind aber außerhalb der Kreditwirtschaft nicht nutzbar.

Man erkennt an diesen wenigen Beispielen, dass zwischen Benutzerfreundlichkeit und Sicherheit abzuwägen ist. Je mehr Sicherheit in ein Authentifikationssystem gesteckt wird, desto niedriger wird die Akzeptanzrate beim Anwender sein. Es bleibt eine der großen Herausforderungen, ein sicheres Authentifikationsverfahren zu finden, das gleichzeitig eine hohe Nutzerakzeptanz hat. Dabei will der Benutzer stets neue Anwendungen mit einfachem User-Interface haben, ist aber weiterhin besorgt über steigende Gefahren ${ }^{3}$. Die wachsende Mobilität der Anwender ist ein weiterer wichtiger Faktor. Die Benutzer wollen Zugang zu den Anwendungen nicht nur von ihrem heimischen Computer aus, sondern auch aus dem Büro, während des Urlaubs mit Smartphone oder Tablet.

Tatsächlich ist es heute so, dass der Nutzer eine Vielzahl technisch unterschiedlich ausgeprägter Authentifikationsverfahren mit Bindung an einen Geschäftspartner - unterstützen muss, was die Convenience, und letztlich auch die Bereitschaft zur tatsächlichen Nutzung, negativ beeinflusst. Dies hat die Industrie erkannt und sich z. B. im Rahmen der FIDO (Fast IDentity Online) Allianz zusammengeschlossen. FIDO hat das Ziel, die fehlende Interoperabilität zwischen den zum Zweck der starken Authentifikation eingesetzten Geräten zu beseitigen. Dazu entwickelt die Allianz Spezifikationen mit dem Anspruch offene, skalierbare und interoperable Mechanismen zur sicheren Authentifikation von Online-Nutzern zur Verfügung zu stellen.

Es bleibt abzuwarten, wie künftige Entwicklungen für sichere und handhabbare Authentifikationen vom Markt angenommen werden - benötigt werden sie auf alle Fälle.

\footnotetext{
1 Warum die elD-Funktion des neuen Personalausweises (noch) keinen Erfolg hat, Dr. Christian Hoffmann, www.government2020.de

2 Mit Ausnahme des HBCI Standard (Home Banking Computer Interface), der als DK-weiter Standard von allen Banken und Sparkassen unterstützt wird.

3 Bitkom, 22.09.2015, Internetnutzer gehen pragmatisch mit Datenschutz um; „67 Prozent der Internetnutzer verzichten bewusst auf bestimmte Online Dienste, wenn sie dort persönliche Daten ... angeben müssen" (www.bitkom.org/ Presse/)
} 